\section{Cool radlators}

P. R. Berger et al. (Appl. Phys. Lett. 59, $117-119 ; 1991$ ) have succeeded in combining in a single chip the elements of a laser and an electrical thermostat operating on the Peltier effect (the reverse of the thermoelectric effect by which currents are produced from a temperature gradient). In part this is to stabilize the laser's emission frequency, which depends on its temperature. But

Berger et al. show that varying the temperature with the internal cooler also tunes the frequency over a range of 12 $\AA$. They envisage that such tunable laser chips could be used in high-rate data networks, optical logic switches and spectroscopy.

\section{Glue worms}

ENVIRONMENT-conscious do-it-yourself enthusiasts searching for the ultimate superglue need look no further. For there is a glue that withstands pressures of 260 atmospheres, temperatures of $250^{\circ} \mathrm{C}$ and sulphurous stews of toxic chemicals - and it is perfectly natural. It is the collagen that keeps deep-sea hydrothermal vent worms from falling apart. But as F. Gaill et al. show (J. molec. Biol. 221, 209-223; 1991) there is much to learn about the properties of worm glue. Large amounts of the amino acid hydroxyproline are often thought to confer heat resistance on collagen, but little is present in the remarkably heat-stable collagens from the giant vestimentiferan worm Riftia pachyptila and the annelid Alvinella pompejana. Perhaps, the authors suggest, there is too little oxygen in the abyss to sustain the hydroxylation of proline residues, or perhaps the high pressure is responsible.

\section{No nothing}

WHILE many nuclear laboratories are pursuing the newly identified 17-keV neutrino, K. You et al. have been patiently trying to make no neutrinos at all (Phys. Lett. B265, 53-56; 1991). Like those embarked on searches for the exotic heavy variety, these researchers are hoping to reveal new subtleties of the nuclear weak force. The typical source of neutrinos is nuclear $\beta$-decay in which one neutrino and one electron is born. But, depending on whether neutrinos are their own antiparticles or not (whether they are 'Majorana' or 'Dirac' particles), it may be possible for nuclei to decay by the emission of two electrons accompanied by no neutrinos. Searches for such decays have so far been unsuccessful. You et al. realized that the nuclide ${ }^{48} \mathrm{Ca}$ should give a distinct signature and so increase their chance of success. Nevertheless, after watching $43 \mathrm{~g}$ of the isotope in $37 \mathrm{~kg}$ of natural $\mathrm{CaF}_{2}$ crystals for 7,588 hours in the bottom of a coalmine, they are still waiting for the first event. cortical representation of the digits - a single digit can occupy an area as large as the entire arm and hand representation in a monkey. If one of the four digital nerves is cut, plasticity is consistent with the underlying spread of thala mocortical afferents described in other species $^{9}$. If all digital nerves are cut, however, a distinctly different type of response is unmasked. In the short term, this takes the form of unusual off- and inhibitory-responses to stimulation of other digits, as well as the presence of many unresponsive neurons. But in longer term experiments the percentage of typical responses increases ${ }^{10}$. The source of these responses seems to be existing connections from other somatosensory areas of cortex which do not have the same degree of precision in the topographic representation of the body surface topography as the primary field.

In monkeys, corticocortical inputs to primary somatosensory cortex are extensive and are not as topographically precise as are inputs from the thalamus. If MOLECULAR FILMS

\section{Laying down organic metals}

\section{Martin R. Bryce}

OVER the past two decades, molecular charge-transfer salts, which conduct electricity (organic metals) have been intensively studied in the form of single crystals $^{1}$. But, as discussed at a recent meeting* , these materials have now been fabricated into thin films by vapour deposition techniques without losing any of their remarkable properties. In this form, they will be much easier to exploit in electronic applications.

A charge-transfer (CT) salt is formed by combination of an electron-donor molecule with an acceptor molecule. Traditionally these salts are electrical insulators but many systems are now known in which stacking of the ionized molecules in the crystal lattice occurs in such a way that extensive electron delocalization can occur. The result is high electronic conductivity at room temperature, $\sigma_{\mathrm{rt}}=10-1,000 \mathrm{~S} \mathrm{~cm}^{-1}$ (for comparison the conductivity of copper metal is $10^{6} \mathrm{~S} \mathrm{~cm}^{-1}$ ), and even bulk superconductivity at very low temperatures (below 12 $\mathrm{K}$ ) in a few cases. The donor molecules are typically planar (or nearly planar) sulphur heterocycles which have low ionization potentials, including TTF or BEDT-TTF (see figure), and the counterion is an inorganic anion, such as $\mathrm{I}_{3}^{-}, \mathrm{PF}_{6}^{-}, \mathrm{ReO}_{4}^{-}$or a more exotic species, such as $\mathrm{Cu}\left[\mathrm{N}(\mathrm{CN})_{2}\right] \mathrm{Br}$ (ref. 2). A striking difference between these organic metals and inorganic metals is that the

* Molecular Conductors for Information Technology, Sesimbra, Portugal, 23-25 May 1991. corticocortical connections are responsible for the unexpected plasticity shown by the Taub monkeys, then it will still be possible to hold to the idea that adult brains are the stable product of their development - and avoid the inference that the generation of new neuronal projections is involved.

Mike Calford is at the Vision, Touch and Hearing Research Centre, University of Queensland, Queensland, 4072 Australia.

1. Pons, T. P. et al. Science 252, 1857-1860 (1991)

2. Kaas, J. H. A. Rev. Neurosci. 14, 137-167 (1991).

3. Garraghty, P. E. \& Sur, M. J. comp. Neurol. 294 583-593 (1990)

. Dykes, R. W., Landry, P., Metherate, R. \& Hicks, T. P. J. Neurophysiol. 52, 1066-1093 (1984).

5. Calford, M. B. \& Tweedale, R. J. Neurophysiol. 65. 178-187 (1991).

6. Calford, M. B. \& Tweedale, R. Science 249, 805-807 (1990)

7. Calford, M. B. \& Tweedale, R. Nature 332, 446-448 (1988).

8. Devor, M. \& Wall, P. D. J. Neurosci. 1, 679-684 (1981)

9. Turnbull, B. G. \& Rasmusson, D. D. Somatosens. Motor Res. 7, 365-389 (1990)

10. Rasmusson, D. D. J. comp. Neurol. 205, 313-326 (1982) former are highly anisotropic; that is, high conductivity occurs along only one (or occasionally two) of the crystallographic axes.

For the many technical applications envisaged for organic metals, far more attention has been given to electrically conducting polymers (polyacetylene and polyaniline, for example), which are relatively easy to prepare in the form of free-standing thin films $\mathrm{s}^{3,4}$, than to CT salts. Rechargeable solid-state batteries, electrolytic capacitors and electrochromic devices, such as 'smart windows' have been developed using these polymers. The frailty of the crystals of molecular CT salts has imposed a severe limitation on their practical usage. It is recognized, however, that if this problem can be overcome, CT salts may offer distinct advantages over polymers. First, there is greater scope for fine-tuning their properties by chemical modification of the starting materials. Second, CT salts are usually stable in air, in contrast to many conducting polymers which readily decompose. And lastly, the most glamorous property, organic superconductivity, has been found only in CT salts, not in polymers (presumably this is at least partly because of inherent disorder found in the latter systems). It is, therefore, an exciting step forward that stable thin films of CT salts can now be fabricated using vapour deposition techniques.

Several semiconducting thin films of 